\title{
Characterization of Nanostructured Magnetic Recording Media
}

\author{
J.E. Wittig, J.F. Al-Sharab, J. Bentley*, and N.D. Evans* \\ Dept. of Electrical Engineering, Vanderbilt University, Nashville, TN 37232, USA \\ *Metals \& Ceramics Div., Oak Ridge National Laboratory, Oak Ridge, TN 37831, USA
}

For determining structure-property relationships of longitudinal or perpendicular magnetic recording media with a typical grain size of about $10 \mathrm{~nm}$, atomic-resolution imaging and nanometer-resolution compositional mapping are a powerful combination. Critical microstructural parameters of Co-alloy thin films that influence the magnetic properties include crystalline defects and chemical inhomogenieties. Segregation of elements like $\mathrm{Cr}$ and $\mathrm{B}$ to the grain boundaries in sputtered Coalloy recording media produces a paramagnetic layer that isolates individual magnetic grains by breaking the magnetic exchange. Depletion of $\mathrm{Cr}$ in the grain interiors also affects the magnetic properties by increasing the magnetocrystalline anisotropy, $\mathrm{Ku}$, of the Co-rich magnetic domains. Energy-filtered transmission electron microscopy (EFTEM) elemental maps together with highresolution imaging (HRTEM) have shown that the level of $\mathrm{Cr}$ segregation/depletion is related to the grain boundary character as well as the crystallographic texture of the magnetic layer $[1,2]$. Spectrum imaging using x-ray energy-dispersive spectroscopy (EDS) and parallel electron energyloss spectroscopy (PEELS) is a complementary method to characterize these Co-alloys for the chemical distribution of elements such as Ta, $\mathrm{Pt}$, and $\mathrm{B}[2]$.

In order to correlate the structure and chemical composition, high-resolution images and chemical maps must be obtained from identical areas. Unfortunately, this is not experimentally straightforward because the images and elemental maps are often collected at different magnifications, at slightly different specimen tilts to appropriately control diffracting conditions, in different microscope operational modes, or even with two different microscopes. Figures 1 and 2 are examples of HRTEM images with corresponding EFTEM $\mathrm{Cr} / \mathrm{Co}$ intensity ratio images $\left(\mathrm{Cr} \mathrm{L}_{23}\right.$ core-loss map divided by the Co $\mathrm{L}_{23}$ core-loss map) from $\mathrm{Co}_{80} \mathrm{Cr}_{16} \mathrm{Ta}_{4}$ and $\mathrm{Co}_{84} \mathrm{Cr}_{16}$ magnetic thin films. These magnetic layers (25 nm thick) were D.C. magnetron sputtered onto identical CrMo seedlayers at a $250^{\circ} \mathrm{C}$ substrate temperature. The $\mathrm{BCC}$ CrMo grows with a strong [001] crystallographic texture that produces a Co [11-20] growth direction with the c-axis in the plane of magnetic thin film such that $(0002)_{\mathrm{Co}} / /(110)_{\mathrm{Cr}}$. Plan-view samples for TEM were prepared by backthinning with the final ion milling performed with a single gun at $4 \mathrm{keV}$ and $12^{\circ}$. The HRTEM was performed with a Philips CM200FEG and the EFTEM with a Philips CM30 (LaB 6 cathode) and a Gatan Imaging Filter (GIF)[3]. Careful documentation of the area imaged in the CM200 allowed the same area to be analyzed by EFTEM. Matching areas required image rotation and image resizing to produce equal magnification and superposition of the HRTEM images and elemental maps.

A comparison of Figures 1 and 2 reveals the strong influence of Ta on both the structure and chemical inhomogenieties of the Co-alloy magnetic layer. The randomly oriented grain boundaries in $\mathrm{Co}_{80} \mathrm{Cr}_{16} \mathrm{Ta}_{4}$ appear amorphous in the HRTEM image and are accompanied by well-defined $\mathrm{Cr}$ grain boundary segregation. In contrast, the structure of the binary $\mathrm{Co}_{84} \mathrm{Cr}_{16}$ exhibits numerous $90^{\circ}$ grain boundaries (c-axes at $90^{\circ}$ produced by two orthogonal variants nucleated on a single seedlayer grain) with a corresponding complicated $\mathrm{Cr}$ segregation distribution. Although both the $\mathrm{Co}_{80} \mathrm{Cr}_{16} \mathrm{Ta}_{4}$ and $\mathrm{Co}_{84} \mathrm{Cr}_{16}$ were sputtered onto CrMo seedlayers with essentially the same grain size, we believe the binary $\mathrm{Co}_{84} \mathrm{Cr}_{16}$ alloy grew from multiple nucleation sites on each CrMo underlayer grain and developed a more island-like growth mode, whereas the $\mathrm{Co}_{80} \mathrm{Cr}_{16} \mathrm{Ta}_{4}$ grew with a more layer-bylayer growth mechanism. The proposed difference in growth mode is related to a difference in the interfacial energy between the Co alloys and the CrMo underlayer. Quantitative analysis shows that $4 \% \mathrm{Ta}$ also results in increased grain-boundary $\mathrm{Cr}$ segregation, on the average $50 \%$ more than for the binary material. Improved grain boundary isolation is partially responsible for the greater coercivity (Hc) of 1670 Oe for $\mathrm{Co}_{80} \mathrm{Cr}_{16} \mathrm{Ta}_{4}$ compared to only 724 Oe for the $\mathrm{Co}_{84} \mathrm{Cr}_{16}$ film [4]. 


\section{References}

[1] J.E. Wittig, J.F. Al-Sharab, J. Bentley, and N.D. Evans, pp. 429-432 in Inst. Phys. Conf. Ser. No 168, Proc. EMAG2001 (Dundee), IOP Publishing, Bristol, 2001.

[2] J.E. Wittig et al., Scripta Mater. 48 (2003) 943.

[3] J.E. Wittig, J. Bentley, and T.P. Nolan, Mater. Res. Soc. Symp. Proc., 562 (1999) 3.

[4] Financial support from the Information Storage Industry Consortium is gratefully acknowledged. Research at the Oak Ridge National Laboratory SHaRE Collaborative Research Center was sponsored by the Division of Materials Sciences and Engineering, U.S. Department of Energy, under contract DE-AC05-00OR22725 with UT-Battelle, LLC.
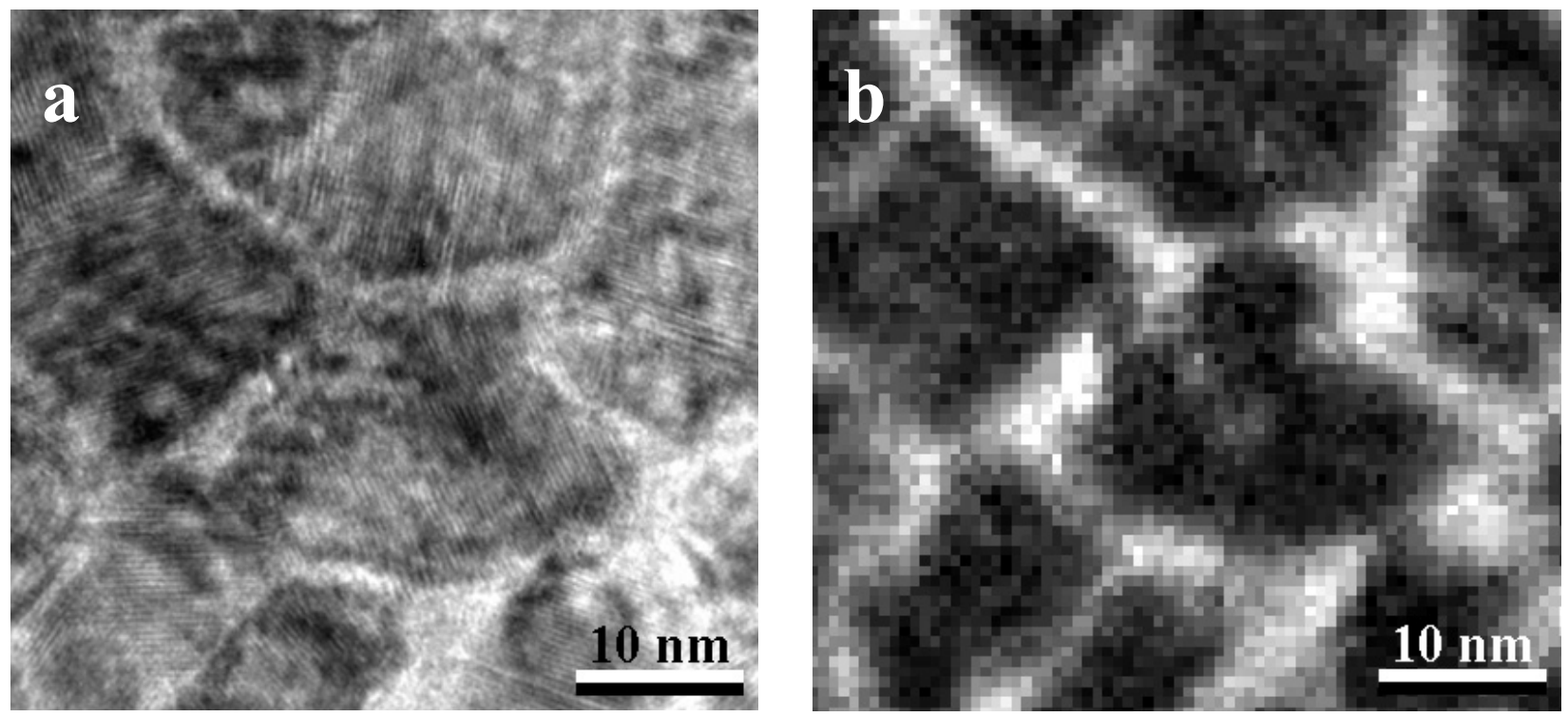

Figure 1 - (a) HRTEM image and (b) EFTEM Cr/Co intensity ratio image of $\mathrm{Co}_{80} \mathrm{Cr}_{16} \mathrm{Ta}_{4}$
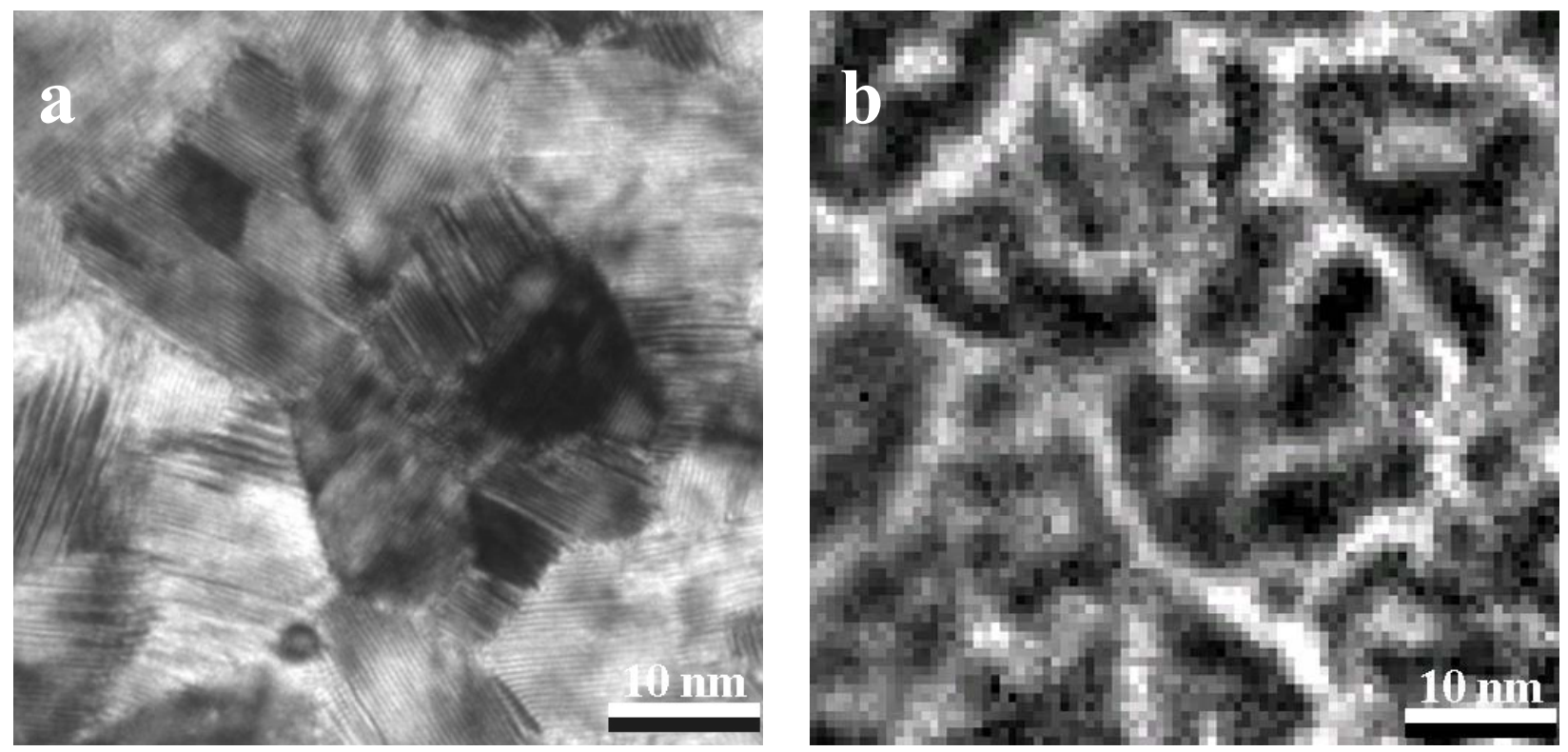

Figure 2 - (a) HRTEM image and (b) EFTEM Cr/Co intensity ratio image of $\mathrm{Co}_{84} \mathrm{Cr}_{16}$. 